\title{
Reaction dynamics of exotic and stable weakly-bound nuclei using a four- body continuum-discretized coupled-channels formalism
}

\author{
Manuela Rodríguez-Gallardo ${ }^{1,2, *}$ and Jesús Casal $^{1}$ \\ ${ }^{1}$ Departmento de Física Atómica, Molecular y Nuclear, Universidad de Sevilla, Spain \\ ${ }^{2}$ Instituto Carlos I de Física Teórica y Computacional, Universidad de Sevilla, Spain
}

\begin{abstract}
Reactions induced by four weakly-bound nuclei on a lead target, at energies around the Coulomb barrier, are described using the same four-body continuum-discretized coupled-channels formalism. The nuclei considered are the halo nuclei ${ }^{11} \mathrm{Li}$ and ${ }^{6} \mathrm{He}$, the stable nucleus ${ }^{9} \mathrm{Be}$ and the proton-rich nucleus ${ }^{10} \mathrm{C}$. The differences and similarities on the reaction dynamics are addressed.
\end{abstract}

\section{Introduction}

A unified description of the structure and reactions, for the all nuclei at the Segrè chart, is still a problem to be solved in nuclear physics [1]. Weakly-bound nuclei can be the nexus between stable and exotic nuclei. Weaklybound (stable or exotic) nuclei have a low breakup threshold, making likely the breakup of the system when colliding with another nucleus.

Among weakly-bound nuclei, an interesting case is the so-called Borromean nuclei, i.e. three-body composite systems with no binary bound states. With this structure, halo nuclei such as ${ }^{11} \mathrm{Li}$ and ${ }^{6} \mathrm{He}$ are weakly-bound, spatially extended systems, comprising a core and two valence nucleons. On the other hand, the nucleus of ${ }^{9} \mathrm{Be}$ is a stable weakly-bound nucleus with a Borromean structure consisting of two alpha particles and one weakly-bound neutron. Going a step further, the Brunnian nucleus ${ }^{10} \mathrm{C}$ has a four-body structure with no bound binary subsystems. This nucleus can be studied approximately in a three-body model as ${ }^{8} \mathrm{Be}+p+p$.

The description of the reactions induced by these four nuclei, in order of increasing separation energy and using the same formalism, is the objective of this work. For that, the reactions on a same target, ${ }^{208} \mathrm{~Pb}$, at energies around the Coulomb barrier for each system are considered. The formalism used to study these reactions is the four-body continuum-discretized coupled-channels formalism $[2,3]$.

In Sec. 2 we introduce the continuum-discretized coupled-channels formalism. In Sec. 3 we compare the theoretical results with the available experimental data for the reaction of ${ }^{11} \mathrm{Li},{ }^{6} \mathrm{He},{ }^{9} \mathrm{Be}$ and ${ }^{10} \mathrm{C}$ on ${ }^{208} \mathrm{~Pb}$ at energies around the Coulomb barrier. Finally in Sec. 4 we present the main conclusions.

*e-mail: mrodri@us.es

\section{Continuum-Discretized Coupled-Channels (CDCC) formalism}

The coupling to breakup channels in reactions involving weakly-bound nuclei can be formally described within the CDCC formalism [4], in which the total scattering wave function is expanded in internal states of the projectile in a given few-body model. This is typically referred to as three-body CDCC, for two-body projectiles [4], or fourbody CDCC, for three-body projectiles [2, 5]. The corresponding coupling form factors are generated following a multipole expansion of the projectile-target interaction, using as input suitable fragment-target optical potentials. Details of this procedure can be found in Eqs. (12-14) of Ref. [2]. Each term of the expansion is referred to as a multipole of order $Q$.

In the present work, for ${ }^{11} \mathrm{Li},{ }^{6} \mathrm{He},{ }^{9} \mathrm{Be}$ and ${ }^{10} \mathrm{C}$ nuclei induced reactions we perform four-body CDCC calculations using the three-body structure models $\left({ }^{9} \mathrm{Li}+n+n\right.$, $\alpha+n+n, \alpha+\alpha+n$ and $\left.{ }^{8} \mathrm{Be}+p+p\right)$ and fragment-target optical potentials presented in Refs. [3, 6-8]. These reaction calculations have no free-parameters, once the projectile structure is adjusted to reproduce the experimentally known energy spectrum.

The states of ${ }^{11} \mathrm{Li}$ and ${ }^{6} \mathrm{He}$ are computed using a binning procedure (Ref. [3]) from the true three-body continuum states, whereas ${ }^{9} \mathrm{Be}$ and ${ }^{10} \mathrm{C}$ states are generated using a pseudostate approach, the analytical Transformed Harmonic Oscillator method presented in Ref. [9]. Both procedures use hysperspherical coordinates for describing the projectile nucleus. The binning procedure provides a more precise description of the low-lying continuum and this is essential for halo nuclei $\left({ }^{6} \mathrm{He},{ }^{1} 1 \mathrm{Li}\right)$. Nevertheless, the implementation of the binning procedure for three-body systems and, in particular, for three-body nuclei with more than one charged particle is complicated. For that reason, a pseudostate approach is assumed for ${ }^{9} \mathrm{Be}$ and ${ }^{10} \mathrm{C}$, providing converged results for the reactions considered. 
Once the projectile structure is obtained, the CDCC equations are solved up to convergence in the number of partial waves and excitation energy above the respective breakup thresholds, including continuum couplings.

Within the CDCC formalism, the influence of the projectile binding energy on the reaction dynamics is implicitly contained in the corresponding coupling potentials. If the ground state is closer to the threshold, its wave function explores larger distances, increasing the coupling to the low-lying continuum states. This, in first order, produces larger breakup cross sections as less bound is the system.

\section{Results}

Elastic differential cross sections for the reactions ${ }^{11} \mathrm{Li}+{ }^{208} \mathrm{~Pb}$ at $29.8 \mathrm{MeV},{ }^{6} \mathrm{He}+{ }^{208} \mathrm{~Pb}$ at $22 \mathrm{MeV},{ }^{9} \mathrm{Be}+{ }^{208} \mathrm{~Pb}$ at $44 \mathrm{MeV}$ and ${ }^{10} \mathrm{C}+{ }^{208} \mathrm{~Pb}$ at $66 \mathrm{MeV}$ are studied comparing theoretical calculations and experimental data. For the first two reactions, the differential cross section of fragment $\left({ }^{9} \mathrm{Li} /{ }^{4} \mathrm{He}\right)$ production, for which experimental data exist, are also addressed. The order for presenting the results is starting with the less bound nucleus, ${ }^{11} \mathrm{Li}$, and finishing with the nucleus that is more bound, ${ }^{10} \mathrm{C}$.

\section{$3.1^{11} \mathrm{Li}$}

The Borromean nucleus ${ }^{11} \mathrm{Li}$ is bound only by $0.369 \mathrm{MeV}$ with respect to the ${ }^{9} \mathrm{Li}+n+n$ threshold. To describe the ${ }^{11} \mathrm{Li}$ nucleus, a three-body structure is assumed with an inert and spinless ${ }^{9} \mathrm{Li}$ core surrounded by two valence neutrons. In that way, the $J^{\pi}=3 / 2-$ ground state becomes a $J^{\pi}=0^{+}$ state. For details on the structure calculations, see Refs. [6, 10].

In order to study the reaction of ${ }^{11} \mathrm{Li}$ on ${ }^{208} \mathrm{~Pb}$ target at $29.8 \mathrm{MeV}$, states up to a maximum energy of $5 \mathrm{MeV}$ above the breakup threshold and with angular momenta $J^{\pi}=0^{+}, 1^{-}, 2^{+}, 3^{-}$are included. The elastic differential cross section for this reaction is very sensitive to the $B(E 1)$ strength close to the threshold and, in particular, to the presence of a dipole resonance. Since the precise location of this resonance has not yet been firmly established, we adjusted the position of this resonance to the energy that best reproduces the elastic and breakup cross sections. This gives an energy of $0.32 \mathrm{MeV}$ above the breakup threshold. This value is in agreement with the latest experimental results published Refs. [11, 12].

Fig. 1 shows the elastic differential cross section, relative to Rutherford cross section, as a function of the centerof-mass (c.m.) scattering angle for the reaction of ${ }^{11} \mathrm{Li}$ on ${ }^{208} \mathrm{~Pb}$ at $29.8 \mathrm{MeV}$. Experimental data [10] are plotted by yellow circles. The four-body CDCC calculation including only the ground state is represented by the pink dashed line and the full four-body CDCC calculation is the red solid line. It can be seen that the inclusion of the coupling to the continuum produces a very large reduction of the elastic cross section that describes very well the data. The reduction of the cross section with respect to the Rutherford scattering is attributed to the strong dipole coupling

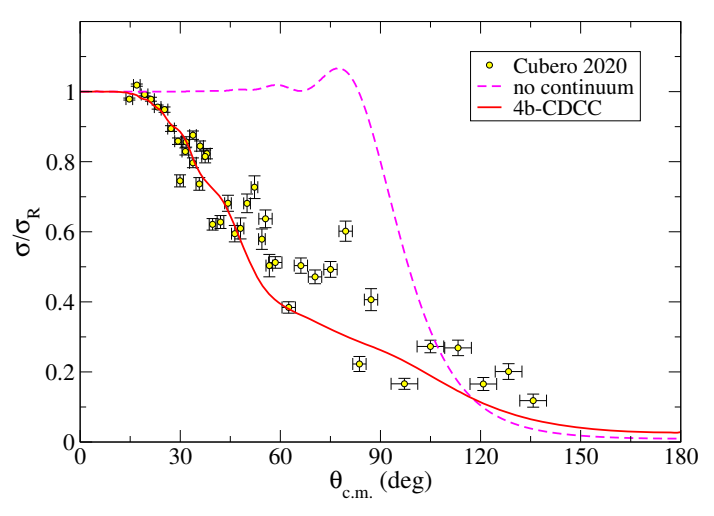

Figure 1. Elastic differential cross section relative to Rutherford as a function of the c.m. scattering angle for the reaction ${ }^{11} \mathrm{Li}+{ }^{208} \mathrm{~Pb}$ at $29.8 \mathrm{MeV}$.

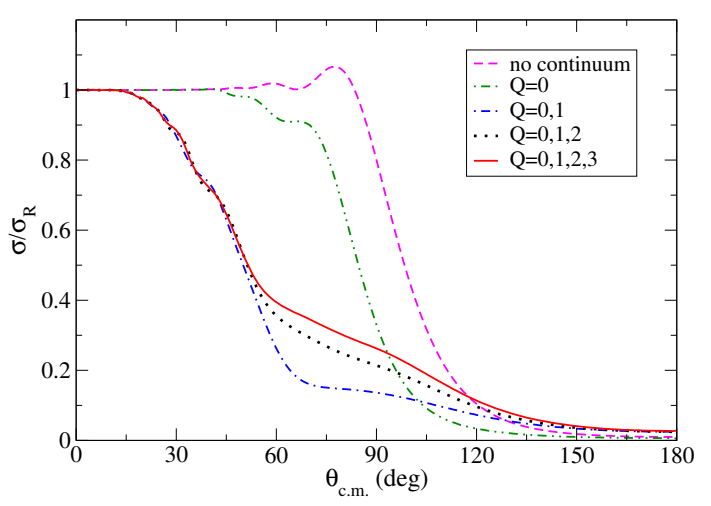

Figure 2. Effect of the different $Q$ multipoles on the elastic differential cross section relative to Rutherford as a function of the c.m. scattering angle for the reaction ${ }^{11} \mathrm{Li}+{ }^{208} \mathrm{~Pb}$ at $29.8 \mathrm{MeV}$.

between the ground and the continuum states in ${ }^{11} \mathrm{Li}$, as it can be seen in Fig. 2. In this figure the contribution of the different multipoles to the elastic differential cross section, with respect to Rutherford cross section, is shown.

Fig. 3 shows differential cross section of ${ }^{9} \mathrm{Li}$ fragments, in the laboratory frame. Experimental data $[6,13]$ are plotted by yellow circles. The four-body CDCC calculation is the red solid line. This calculation reproduces the data for almost the whole angular range. This means that the elastic breakup is the process dominating in this reaction. It is also included in the figure, with a blue dashed line, the contribution from the transfer of the two halo neutrons to the continuum of the target (for details see Ref. [13]). This process gives a relevant contribution for the very backward angles only.

\section{$3.2{ }^{6} \mathrm{He}$}

The Borromean nucleus ${ }^{6} \mathrm{He}$ is bound by $0.973 \mathrm{MeV}$ with respect to the ${ }^{4} \mathrm{He}+n+n$ threshold. Apart from the ground state, with angular momentum $J^{\pi}=0^{+}$, a $2^{+}$resonance is experimentally known at $0.825 \mathrm{MeV}$ over the breakup threshold. To describe the ${ }^{6} \mathrm{He}$ nucleus, a three-body structure is assumed with an inert ${ }^{4} \mathrm{He}$ core surrounded by two 


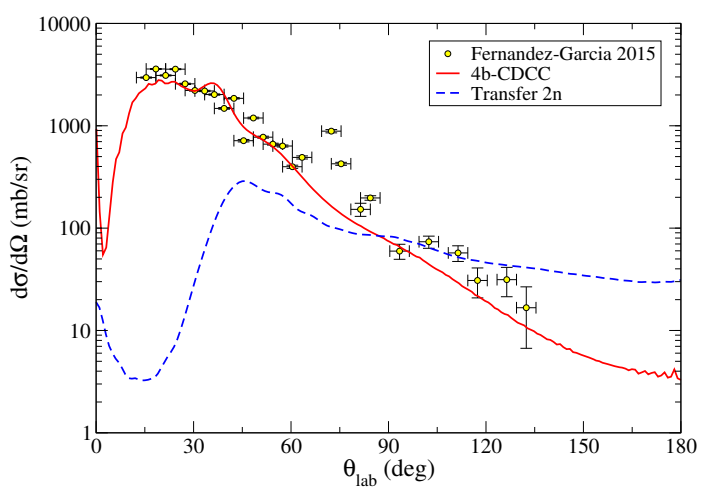

Figure 3. Differential cross section of the ${ }^{9} \mathrm{Li}$ fragments, in the laboratory frame, for the reaction ${ }^{11} \mathrm{Li}+{ }^{208} \mathrm{~Pb}$ at $29.8 \mathrm{MeV}$.

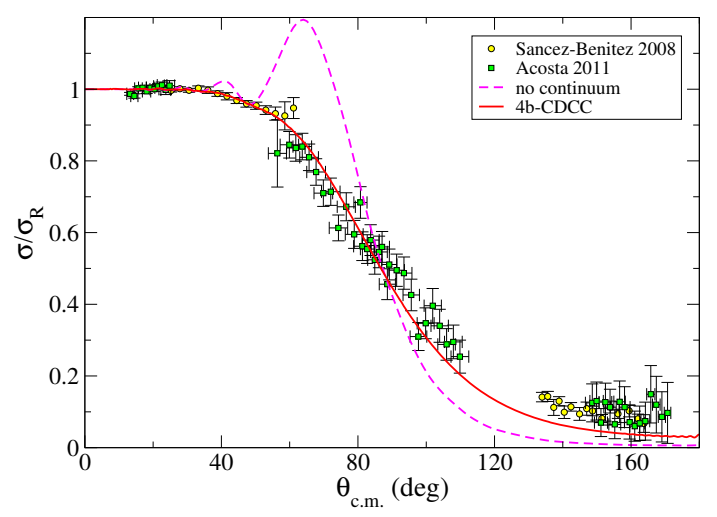

Figure 4. Elastic differential cross section, relative to Rutherford, as a function of the c.m. scattering angle for the reaction ${ }^{6} \mathrm{He}+{ }^{208} \mathrm{~Pb}$ at $22 \mathrm{MeV}$.

valence neutrons. For details on the structure calculations see Ref. [3].

In order to study the reaction of ${ }^{6} \mathrm{He}$ on ${ }^{208} \mathrm{~Pb}$ target at $22 \mathrm{MeV}$, states up to $8 \mathrm{MeV}$ above the breakup threshold and with angular momenta $J^{\pi}=0^{+}, 1^{-}, 2^{+}$are included. The elastic cross section angular distribution with respect to Rutherford is shown in Fig. 4. The experimental data are plotted by yellow circles [14] and green squares [15]. The pink dashed line is the four-body CDCC calculation without including the continuum and the red solid line is full four-body CDCC calculation. As in the ${ }^{11} \mathrm{Li}$ case, the inclusion of the continuum part of the spectrum produces an important, although smaller, reduction of the elastic cross section that describes very well the data. In particular, the rainbow at around 60 degrees disappears. This reduction is due mostly to the coupling to the dipolar states, although in the ${ }^{6} \mathrm{He}$ there is no a fully-fledged dipolar resonance. The effect of the different multipoles to the elastic distribution is shown in Fig. 5.

Fig. 6 shows the differential cross section of $\alpha$-particle production, in the laboratory frame, for the reaction of ${ }^{6} \mathrm{He}$ on ${ }^{208} \mathrm{~Pb}$ at $22 \mathrm{MeV}$. Experimental data from two experiments are plotted by yellow circles [16] and green squares [15]. The red solid line is the full four-body CDCC calculation and the blue dashed line is a calculation con-

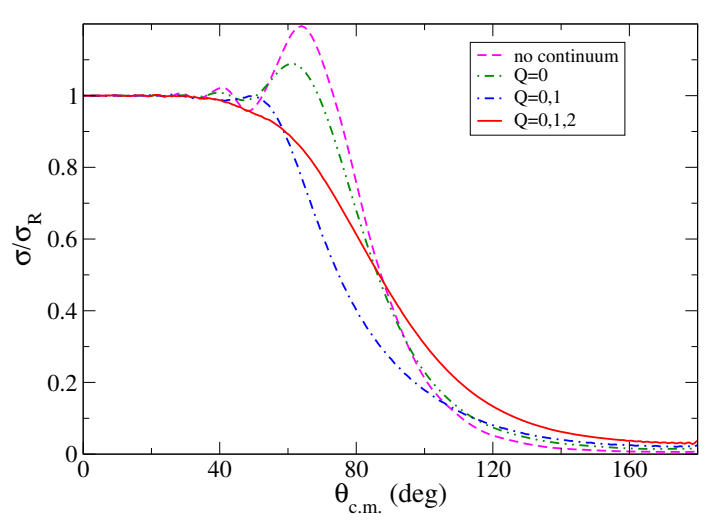

Figure 5. Effect of the different $Q$ multipoles on the elastic differential cross section, relative to Rutherford, as a function of the c.m. scattering angle for the reaction of ${ }^{6} \mathrm{He}$ on ${ }^{208} \mathrm{~Pb}$ at $22 \mathrm{MeV}$.

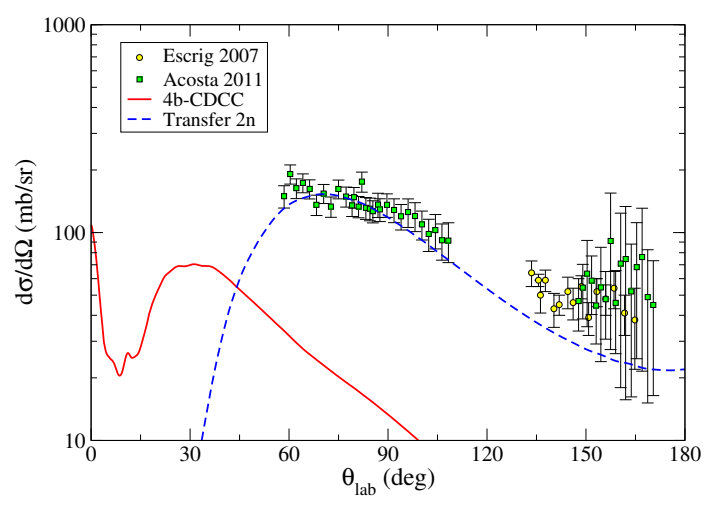

Figure 6. Differential cross section of $\alpha$-particle production, in the laboratory frame, for the reaction ${ }^{6} \mathrm{He}+{ }^{208} \mathrm{~Pb}$ at $22 \mathrm{MeV}$.

sidering a scheme in which the two halo neutrons are transferred to the continuum of the target (see [16] for details). Contrary to the ${ }^{11} \mathrm{Li}$ case, the elastic breakup (obtained from CDCC) only dominates the angular region below 30 degrees. In the region where experimental data are available (above 30 degrees) the transfer of the two halo neutrons to the target is the process that dominates this reaction. This process is one of the contributions to the nonelastic breakup [17].

\section{$3.3{ }^{9} \mathrm{Be}$}

The Borromean nucleus ${ }^{9} \mathrm{Be}$ is bound by $1.5736 \mathrm{MeV}$ with respect to the ${ }^{4} \mathrm{He}+{ }^{4} \mathrm{He}+n$ threshold. The low-energy spectrum of ${ }^{9} \mathrm{Be}$ shows, apart from the $J^{\pi}=3 / 2^{-}$ground state, several resonances with angular momenta $J^{\pi}=$ $1 / 2^{+}, 5 / 2^{-}, 1 / 2^{-}, 5 / 2^{+}, 3 / 2^{+}$, and possibly others, whose positions and widths have been investigated by many authors in different experiments [18-20]. The spin-parity assignment for some of these states is still under discussion, although there are no implications for the lowest $1 / 2^{+}$and $5 / 2^{-}$resonances, that are fairly-well reproduced in the theoretical model. Details on the structure calculations can be found in Ref. [7].

In order to study the reaction of ${ }^{9} \mathrm{Be}$ on ${ }^{208} \mathrm{~Pb}$ target at $44 \mathrm{MeV}$, states up to $8 \mathrm{MeV}$ above the breakup threshold 


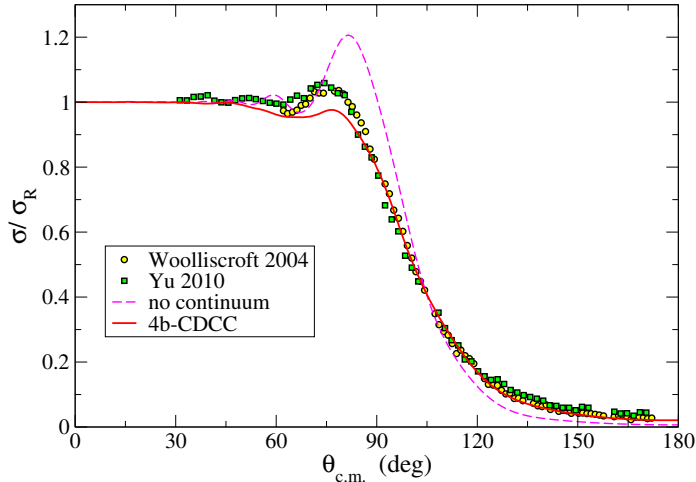

Figure 7. Elastic differential cross section, relative to Rutherford, as a function of the c.m. scattering angle for the reaction ${ }^{9} \mathrm{Be}+{ }^{208} \mathrm{~Pb}$ at $44 \mathrm{MeV}$.

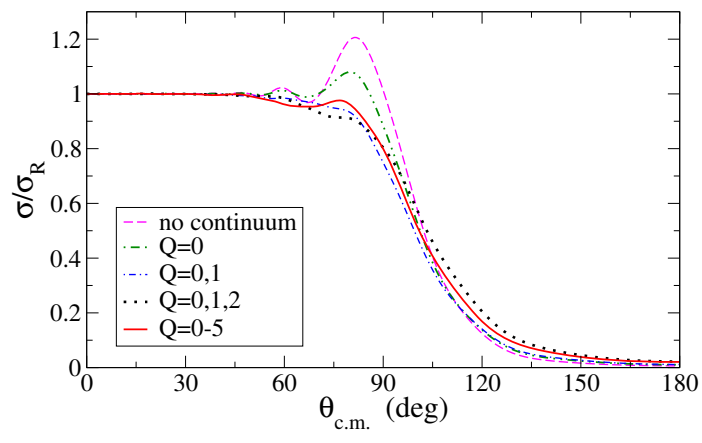

Figure 8. Effect of the different $Q$ multipoles on the elastic differential cross section, relative to Rutherford, as a function of the c.m. scattering angle for for the reaction ${ }^{9} \mathrm{Be}+{ }^{208} \mathrm{~Pb}$ at $44 \mathrm{MeV}$.

and with angular momenta $J^{\pi}=1 / 2^{ \pm}, 3 / 2^{ \pm}, 5 / 2^{ \pm}$are included. The elastic differential cross section, with respect to Rutherford, as a function of the c.m. scattering angle is shown in Fig. 7. The experimental data are plotted by yellow circles [21] and green squares [22]. The pink dashed line is the four-body CDCC calculation without including the continuum and the red solid red is the full four-body CDCC calculation. It can be seen that the coupling to the continuum is still important to reproduce the experimental data, specially the effect of decreasing the rainbow at 80-90 degrees.

Fig. 8 shows the contribution of the different multipoles to the elastic differential cross section. In this case, the effect of the monopolar and dipolar contributions are of the same order of magnitude. This difference with respect to the halo nuclei, ${ }^{6} \mathrm{He}$ and ${ }^{11} \mathrm{Li}$ can be clearly seen comparing Figs. 2, 5 and 8 at the rainbow area for each reaction.

\section{$3.4{ }^{10} \mathrm{C}$ induced reactions}

The Brunnian nucleus ${ }^{10} \mathrm{C}$ is bound by $3.820 \mathrm{MeV}$ with respect the ${ }^{4} \mathrm{He}+{ }^{4} \mathrm{He}+p+p$ threshold. The ground state is a $J^{\pi}=0^{+}$state and a $2^{+}$excited bound state appears close to the breakup threshold. Here, ${ }^{10} \mathrm{C}$ is described within a ${ }^{8} \mathrm{Be}+p+p$ configuration. Details of the structure calculation can be found in Ref. [8].

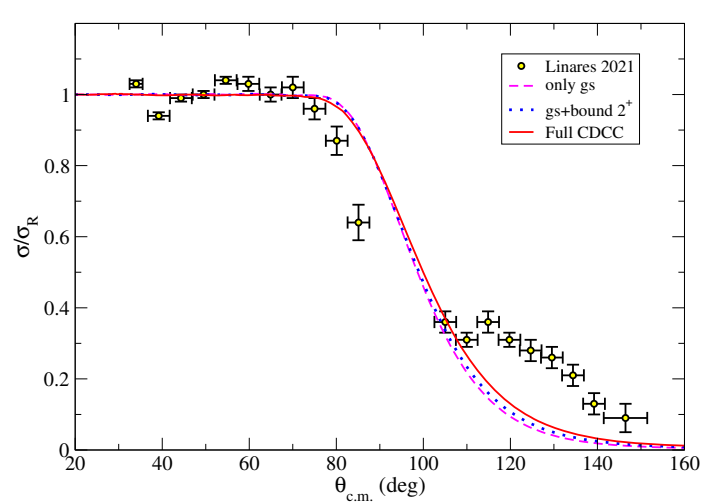

Figure 9. Elastic differential cross section, relative to Rutherford, as a function of the c.m. scattering angle for the reaction of ${ }^{10} \mathrm{C}+{ }^{208} \mathrm{~Pb}$ at $66 \mathrm{MeV}$.

In order to study the reaction of ${ }^{10} \mathrm{C}$ on ${ }^{208} \mathrm{~Pb}$ target at $66 \mathrm{MeV}$, states up to $8 \mathrm{MeV}$ above the breakup threshold and with angular momenta $J^{\pi}=0^{+}, 1^{-}, 2^{+}$are included. The elastic cross section with respect to Rutherford is shown in Fig. 9. The experimental data [8] is plotted by yellow circles. The dashed pink line is the fourbody CDCC calculation including only the ground state, the dotted blue line is the four-body CDCC calculation including the ground state and the bound excited state and the solid red line is the full four-body CDCC calculation. In this case it is observed that the calculation with only the ground state has no rainbow peak and the coupling to the continuum is very small. Even the coupling to the bound excited state hardly affects the elastic cross section.

\section{Conclusions}

The study of the reactions of four weakly-bound nuclei on a lead target at energies around the Coulomb barrier for each system has been presented. The nuclei considered run from exotic nuclei, the halos ${ }^{11} \mathrm{Li}$ and ${ }^{6} \mathrm{He}$, to the stable nucleus ${ }^{9} \mathrm{Be}$ and proton-rich nucleus ${ }^{10} \mathrm{C}$. All the reactions are studied using the four-body CDCC formalism, that reproduces the elastic experimental data for all the cases.

Comparing the four reactions, it can be see how the coupling to the continuum is crucial to reproduce the elastic data for ${ }^{11} \mathrm{Li},{ }^{6} \mathrm{He}$ and even for ${ }^{9} \mathrm{Be}$, decreasing this effect with the binding energy. For the case of ${ }^{10} \mathrm{C}$, the most bound nucleus, the effect is practically negligible. It is also seen that the coupling that produces the reduction of the rainbow is mainly due to dipolar $(Q=1)$ couplings for the halo nuclei ${ }^{11} \mathrm{Li}$ and ${ }^{6} \mathrm{He}$, meanwhile for ${ }^{9} \mathrm{Be}$ monopolar and dipolar coupling effects are of the same order.

Finally, the cross sections of fragment production for the reactions induced by ${ }^{11} \mathrm{Li}$ and ${ }^{6} \mathrm{He}$, have been addressed showing different mechanisms of reaction. For ${ }^{11} \mathrm{Li}$ on ${ }^{208} \mathrm{~Pb}$ at $29.8 \mathrm{MeV}$, the elastic breakup obtained by the four-body CDCC calculations reproduce the experimental distribution for almost the whole angular range. For ${ }^{6} \mathrm{He}$ on ${ }^{208} \mathrm{~Pb}$ at $22 \mathrm{MeV}$, the elastic breakup is only dominating the forward angles, while at angles larger than 
50 degrees the process is dominated by the transfer of the two halo neutrons to the continuum of the target.

\section{Acknowledgments}

This work was supported by the Spanish Ministry of Economy and Competitiveness, the European Regional Development Fund (FEDER), under Project No. FIS201788410-P and by the European Union's Horizon 2020 research and innovation program, under Grant Agreement No. 654002.

\section{References}

[1] M.A.G. Alvarez, M. Rodríguez-Gallardo, J.P. Fernández-García, J. Casal, J.A. Lay, Phys. Rev. C 103, 054614 (2021)

[2] M. Rodríguez-Gallardo, J.M. Arias, J. GómezCamacho, R.C. Johnson, I.J. Thompson, J.A. Tostevin, Phys. Rev. C 77, 064609 (2008).

[3] M. Rodríguez-Gallardo, J.M. Arias, J. GómezCamacho, I.J. Thompson, J.A. Tostevin, Phys. Rev. C 80, 051601(R) (2009).

[4] M. Yahiro, Y. Iseri, H. Kameyama, M. Kamimura, M. Kawai, Prog. Theor. Phys. Suppl. 89, 32 (1986).

[5] T. Matsumoto, T. Egami, K. Ogata, Y. Iseri, M. Kamimura, M. Yahiro, Phys. Rev. C 73, 051602(R) (2006).

[6] J. P. Fernández-García, M. Cubero, M. RodríguezGallardo et al., Phys. Rev. Lett. 110, 142701 (2013).

[7] J. Casal, M. Rodríguez-Gallardo, J.M. Arias, Phys. Rev. C 92, 054611 (2015).
[8] R. Linares, Mandira Sinha, E.N. Cardozo et al., Phys. Rev. C 103, 044613 (2021).

[9] J. Casal, M. Rodríguez-Gallardo, J. M. Arias, Phys. Rev. C 88, 014327 (2013).

[10] M. Cubero, J. P. Fernández-García, M. RodríguezGallardo et al., Phys. Rev. Lett. 109, 262701 (2012).

[11] R. Kanungo, A. Sanetullaev, M. Tanaka et al., Phys. Rev. Lett. 114, 192502 (2015).

[12] M. Tanaka, R. Kanungo, M. Alcorta et al., Phys. Lett. B 774, 268 (2017).

[13] J. P. Fernández-García, M. Cubero, L. Acosta, Phys. Rev. C 92, 044608 (2015).

[14] A.M. Sánchez-Benítez. D. Escrig, M.A.G. Alvarez et al., Nucl. Phys. A 803, 30 (2008).

[15] L. Acosta, A.M. Sánchez-Benítez, M.E. Gomez et al., Phys. Rev. C 84, 044604 (2011).

[16] D. Escrig, A.M. Sánchez-Benítez, A.M. Moro et al., Nucl. Phys. A 792, 2 (2007).

[17] J. Lei, A.M. Moro, Phys. Rev. C 92, 044616 (2015).

[18] K. Sumiyoshi, H. Utsunomiya, S. Goko, and T. Kajino, Nucl. Phys. A 709, 467 (2002).

[19] C.W. Arnold, T.B. Clegg, C. Iliadis et al., Phys. Rev. C 85, 044605 (2012).

[20] O. Burda, P. von Neumann-Cosel, A. Richter, C. Forssén, B.A. Brown, Phys. Rev. C 82, 015808 (2010).

[21] R. J. Woolliscroft, B. R. Fulton, R. L. Cowin et al., Phys. Rev. C 69, 044612 (2004).

[22] N. Yu, H.Q. Zhang, H.M. Jia et al., J. Phys. G: Nucl. Part. Phys. 37, 075108 (2010). 